\title{
Critical appraisal and update on the clinical utility of agomelatine, a melatonergic agonist, for the treatment of major depressive disease in adults
}

This article was published in the following Dove Press journal:

Neuropsychiatric Disease and Treatment

29 October 2009

Number of times this article has been viewed

\author{
Robert H Howland \\ University of Pittsburgh School \\ of Medicine, Western Psychiatric \\ Institute and Clinic, University of \\ Pittsburgh Medical Center, Pittsburgh \\ PA, USA
}

\begin{abstract}
This article describes the pharmacology of the novel atypical antidepressant drug agomelatine, critically reviews and evaluates its clinical use for the treatment of major depression, and suggests areas for further research. Agomelatine is a synthetic analog of the hormone melatonin. It stimulates the activity of melatonin MT1 and MT2 receptors and inhibits the activity of serotonin 5HT-2C receptor subtypes. Three acute trials demonstrated clinically modest, but statistically significant benefits over placebo. Three acute trials did not find agomelatine more effective than placebo. A meta-analysis of these six trials demonstrated a small, statistically significant, marginally clinically relevant difference between agomelatine and placebo. The only placebo-controlled study in elderly patients did not demonstrate a significant benefit for agomelatine. It was more effective than placebo in only one of two relapse prevention studies. Agomelatine was generally well tolerated compared to placebo. Its side-effect profile is different than and compares favorably to other antidepressant drugs. The overall tolerability of agomelatine in head-to-head comparisons was not substantially better than active drug comparators. Agomelatine is contraindicated in patients with impaired liver function and in patients taking drugs that potently inhibit CYP-1A2 metabolic enzymes. Because elevated liver enzymes are common, and there is a rare risk of more serious liver reactions, routine laboratory monitoring of liver function is recommended periodically throughout treatment. Agomelatine does not have clinically significant advantages compared to other antidepressant drugs, and it has certain limitations and disadvantages. Because of its unique pharmacology and relatively benign tolerability profile, however, it may be a useful alternative for patients who do not respond to or cannot tolerate other antidepressant drugs.
\end{abstract}

Keywords: agomelatine, melatonin, serotonin, antidepressant, major depression

\section{Introduction}

The goal of antidepressant drug therapy for major depression should be to achieve full remission, as demonstrated by the absence of significant depressive symptoms along with a complete recovery of social and vocational function. ${ }^{1}$ With any firstchoice antidepressant medication, about $50 \%$ to $70 \%$ of patients will have a significant treatment response (usually defined as a $50 \%$ or greater decrease in depressive symptoms). Of these treatment responders, however, only about one-half to one-third attain a full remission. A significant proportion of depressed patients are therefore left with residual or persistent symptoms despite apparently adequate antidepressant therapy. The failure to achieve remission with antidepressant therapy is associated with an increased risk of relapse or recurrence, higher levels of impaired social
Correspondence: Robert H Howland Associate Professor of Psychiatry, University of Pittsburgh School of Medicine, Western Psychiatric Institute and Clinic, University of Pittsburgh Medical Center, 38I I O'Hara Street, Pittsburgh PA 15213, USA

Tel + I 4I 2-246-5749

Fax + | 4I2-246-5750

Email howlandrh@upmc.edu 
and vocational function, and a worse long-term prognosis. Chronic or recurrent depression is associated with persistent social and vocational disability, an increased risk of suicide, greater medical morbidity and mortality, and higher health care utilization and costs..$^{2-4}$ Patients with depressive symptoms less severe than major depression also suffer from significant impairment. ${ }^{5,6}$

Symptomatic improvement of depression can facilitate the process of functional recovery, thereby reducing disability, and this can help prevent possible complications related to the illness such as substance abuse and suicide. ${ }^{7}$ Complications related to depression also extend to its effect on various medical conditions. Depression worsens the health outcome and functioning of patients with a variety of medical disorders. ${ }^{8}$ For example, heart disease, diabetes, and osteoporosis are significantly worse in depressed compared to nondepressed patients. ${ }^{9-12}$ Having depression also contributes to poor treatment adherence for other medical conditions, and treating depression can improve medical adherence. ${ }^{13-16}$ Depression not only affects existing medical disorders, but can be associated with an increased risk of the later development of some medical conditions. Compared to nondepressed people, depressed patients are more likely to later develop heart disease, diabetes, and osteoporosis. ${ }^{10,17-19}$ Depression also can adversely affect pregnancy outcome (eg, prematurity and low birthweight). ${ }^{20,21}$ Ineffectively or inadequately treating depression may therefore contribute to the substantial morbidity and mortality associated with many medical conditions as well as with depression itself.

Many types of antidepressant drugs are available, but their absolute effectiveness is limited. ${ }^{22}$ Many patients do not respond to available drugs, or they have residual symptoms despite adequate treatment. Some patients respond to medication, but they develop intolerable side effects and stop treatment. For these reasons, new drug therapies are always needed. Even though a new drug may not necessarily be more effective on average compared to available drugs, it might be relatively more efficacious or better tolerated for certain patients. In this paper, I will describe the pharmacology of the novel atypical antidepressant drug agomelatine, critically review and evaluate its clinical use for the treatment of major depression, and outline suggested areas for further research investigating this unique drug. The literature was reviewed for articles relating to agomelatine on MEDLINE, PsychINFO, and Google Scholar using the search terms "agomelatine" or "S-20098." Additional literature potentially related to agomelatine was searched using the terms "melatonin," "ramelteon," or "TAK-375."

\section{Pharmacology and pharmacodynamic profile of agomelatine}

After a drug is administered, it is eventually distributed to its site of action where it interacts with its particular targets. Pharmacodynamics refers to the pharmacological mechanism of action of a drug at its particular targets, which includes its therapeutic effects as well as any adverse effects. Psychotropic drug targets typically are various enzymes, transporters, and receptors that regulate the synthesis, transmission, and degradation of different chemical neurotransmitters in the central nervous system. How a drug will affect patient function overall depends on the net effect of its intended therapeutic use together with any unintended effect on other organ systems throughout the body. ${ }^{23}$

Agomelatine has been extensively investigated in preclinical studies. ${ }^{24}$ It also has been investigated in clinical trials in Europe for the treatment of depression and was first recommended for approval by the European Medicines Agency in November $2008 .{ }^{25-27}$ Clinical trials of agomelatine for depression (3 short-term efficacy and safety trials and 1 longer-term relapse prevention trial) have been conducted in the United States, but the findings from these studies have not been released and it is not yet approved by the US Food and Drug Administration. ${ }^{28}$ Agomelatine is a synthetic analog of the hormone melatonin. ${ }^{29}$

Melatonin is secreted by the pineal gland and normally serves to regulate various circadian (24-hour) rhythms, including sleep-wake cycles. Disturbances in circadian rhythms have been implicated in the development of mood disorders as well as disrupted sleep patterns. ${ }^{30}$ Phase-shift hypotheses suggest that depression results from internal circadian rhythms that are phase delayed (or phase advanced in some patients) relative to external clock time (as exemplified by the light-dark cycle) and to sleep-wake cycles, resulting in a pathological desynchronization of ordinarily closely linked biological rhythms. The central internal (endogenous) circadian rhythm pacemaker (regulating such 24-hour biological cycles as endocrine function and body temperature) is located in the suprachiasmatic nucleus $(\mathrm{SCN})$ of the hypothalamus. ${ }^{31}$ Light influences SCN function via a neural pathway from the retina to the SCN. A pathway from the $\mathrm{SCN}$ to the pineal gland regulates the synthesis and release of the melatonin. It is notable that melatonin is synthesized from the neurotransmitter serotonin. ${ }^{32}$ Melatonin secretion is regulated internally by the normal autonomous activity of 
the SCN as well as externally by light exposure on the retina. Melatonin is effective for circadian rhythm sleep disturbances (eg, associated with jet lag), but it is only modestly effective in the treatment of insomnia unrelated to circadian rhythm sleep disturbances. ${ }^{33}$ It also does not appear to have inherent antidepressant effects.

Agomelatine is a potent melatonin-receptor agonist drug that strongly binds to and stimulates the activity of melatonin MT1 and MT2 receptors, which are localized within the SCN. ${ }^{34}$ Stimulation of MT1 and MT2 receptors has a normalizing effect on disturbed circadian rhythms and disrupted sleep-wake cycles. Agomelatine also is a serotonin-receptor antagonist that binds to and inhibits the activity of serotonin 5HT-2C receptor subtypes, but it does not bind to other serotonin receptor subtypes. 5HT-2C receptor antagonism is associated with antidepressant and antianxiety activity and also increases slow-wave sleep (which is abnormally diminished in depression). ${ }^{35}$ Agomelatine does not directly affect the uptake of serotonin, norepinephrine, or dopamine. By inhibiting 5HT-2C receptors, however, it secondarily increases norepinephrine and dopamine in the frontal cortex of the brain. ${ }^{36}$ This effect might contribute to its antidepressant activity. Agomelatine does not bind to adrenergic, cholinergic, or histamine receptors.

Ramelteon also is a synthetic analog of melatonin. ${ }^{29}$ It is a MT1 and MT2 receptor agonist drug currently approved in the United States, Europe, and elsewhere for the treatment of insomnia characterized by difficulty falling sleep. ${ }^{34}$ Unlike agomelatine, it does not bind to any serotonin receptors and has not been investigated as an antidepressant. ${ }^{24}$ Mirtazapine and some other antidepressant drugs have 5HT-2C receptor blocking effects. ${ }^{37}$ Many of the so-called atypical or second generation antipsychotic drugs also have 5HT-2C receptor blocking effects, ${ }^{37}$ and these drugs are efficacious as augmentation agents when used together with antidepressant drugs for treatment resistant depression. ${ }^{38}$ The pharmacology of agomelatine, with its combined effects at MT1, MT2, and 5HT-2C receptors, is therefore unique and distinct compared to other antidepressant drugs. Sleep EEG studies demonstrate the benefits of agomelatine on sleep in depressed patients (ie, increased slow-wave sleep and sleep efficiency). ${ }^{39}$ Increases in slow-wave sleep (the deepest stage of sleep) typically correlate with subjective daytime reports of having had a good night's sleep and feeling well rested. ${ }^{40}$ Agomelatine also has been shown to influence circadian rhythms in animals and humans. ${ }^{41-43}$ These effects are all consistent with what is expected based on its MT1, MT2, and 5HT-2C receptor pharmacology.
Stress-response systems are abnormally activated in depression, and the chemical effects of stress have damaging effects on nerve cells. ${ }^{44}$ In particular, persistent or excessive levels of the glucocorticoid hormone cortisol ${ }^{45}$ or the excitatory amino acid glutamate, ${ }^{46}$ each of which has normal physiologic functions in the brain, can result in neurotoxicity (nerve cell atrophy or death). Chronic stress can also result in decreased neurogenesis (nerve cell growth). ${ }^{47}$ Brain imaging studies show structural brain changes (eg, atrophy or enlarged ventricles) in patients with mood disorders, and this may partly reflect stress-induced neurotoxicity or the inhibition of neurogenesis. ${ }^{44,48}$ Many antidepressant drugs have the effect of reducing neurotoxicity or enhancing neurogenesis. ${ }^{44}$ Similarly, agomelatine increases neurogenesis in the hippocampus region of the brain and may also have neuroprotective effects. ${ }^{49,50}$ The effects on neurogenesis and neurotoxicity are mediated in part by influencing glutamate release, glucocorticoid receptor gene expression, and various neurotrophic factors. ${ }^{51-55}$

\section{Pharmacokinetic profile of agomelatine}

Pharmacokinetics refers to the absorption, distribution, metabolism, and excretion of drugs in the body. After oral administration, agomelatine is rapidly and well absorbed from the gastrointestinal tract. ${ }^{56}$ Food tends to slow down the absorption of agomelatine, but this effect is not clinically significant.

Regardless of the route of administration, all drugs will enter the systemic circulation and most will reversibly bind to various plasma proteins. Similar to most other psychotropic drugs, agomelatine is highly protein bound (its plasma protein binding is greater than 95\%). ${ }^{57}$ Taking multiple drugs that bind to the same plasma protein sometimes can cause displacement of the protein-bound fraction of a drug, resulting in higher concentrations of the free fraction. Based on in vitro studies, agomelatine does not modify free concentrations of drugs highly bound to plasma proteins, nor do other drugs affect its protein binding. ${ }^{25}$ However, this has not been studied in vivo in humans.

Agomelatine is almost entirely metabolized through the liver, and it undergoes extensive first pass hepatic metabolism. One specific study investigated the influence of liver insufficiency in patients with hepatic cirrhosis on plasma levels of agomelatine. ${ }^{25}$ In patients with mild hepatic impairment, the increase in agomelatine exposure was more than 50 times higher compared with healthy subjects. For patients with moderate hepatic impairment, the exposure was more than 
100 times higher compared with healthy subjects. In addition, because of decreases in plasma proteins, the unbound free fraction of agomelatine was increased in subjects with hepatic insufficiency. The free fraction was approximately twice as great in patients with moderate hepatic impairment.

The major cytochrome P-450 (CYP-450) enzyme involved in the metabolism of agomelatine is CYP-1A2 (accounting for about 90\% of its metabolism), with minor metabolic contributions by CYP-2C9 and CYP-2C19. ${ }^{24}$ Agomelatine has at least four main metabolites. The pharmacological activity of the metabolites for 5HT-2C, MT1, and MT2 receptors is not clearly established. None of the metabolites have any known toxic effects. Agomelatine does not appear to inhibit or induce the activity of any CYP-450 enzymes in humans, but enzyme induction has been demonstrated in animal studies. ${ }^{25}$

Agomelatine and its metabolites are mainly excreted through the kidneys. The elimination half-life of agomelatine is very short (about 2-3 hours). The effects of renal function on agomelatine pharmacokinetics were investigated in a study of healthy subjects and patients with severe impaired renal function. ${ }^{25}$ In the renal impairment patients, exposure to agomelatine increased more than $25 \%$ compared to healthy subjects.

In humans, the oral bioavailability of agomelatine at doses of $25 \mathrm{mg}$ and $50 \mathrm{mg}$ is very low. The bioavailability may increase at higher doses, perhaps due to saturation of first pass hepatic metabolism or due to nonlinear pharmacokinetics. The oral bioavailability of agomelatine is estimated to be relatively higher in women compared to men and to be relatively higher in elderly versus younger individuals, perhaps due to gender and age effects on hepatic blood flow and metabolic enzyme activity. ${ }^{25}$ However, because of significant intra-individual and inter-individual pharmacokinetic variability, dose changes based on age or gender are not considered to be routinely necessary. Also, bioavailability is relatively higher in nonsmokers versus smokers and in women taking estrogen-containing drugs compared to women who do not. These findings are explained by the metabolic enzyme-inducing effects of smoking and the enzyme-inhibiting effects of estrogens. Agomelatine does not affect the pharmacokinetics of the bronchodilator drug theophylline, which is a substrate for CYP-1A2. The selective serotonin reuptake inhibitor (SSRI) drug fluvoxamine is a potent inhibitor of CYP-1A2 and a moderate inhibitor of CYP-2C9, and it can significantly increase serum concentrations of agomelatine. By contrast, the SSRI drug paroxetine is a moderate inhibitor of CYP-1A2 and does not significantly increase the concentration of agomelatine. The antifungal drug fluconazole is a potent inhibitor of CYP-2C9, but it has not been shown to significantly influence the pharmacokinetics of agomelatine. Specific drug-drug interaction studies involving lithium, lorazepam, alcohol, and valproic acid have not demonstrated any significant effects of these drugs on the pharmacokinetics of agomelatine.

\section{Short-term clinical studies of agomelatine for major depression}

The effectiveness of agomelatine for major depression has been investigated in 3 published $^{58-60}$ and 3 unpublished $^{25}$ randomized, double-blind, placebo-controlled pivotal studies (see Table 1). Paroxetine or fluoxetine was included in some of these trials as an active comparison drug according to European regulatory guidelines. For each of the pivotal studies, the 17-item Hamilton Depression Rating Scale (HDRS) was the primary outcome measure. The primary outcome efficacy variable was the HDRS score for agomelatine versus placebo at endpoint. Response and remission rates based on the HDRS were considered secondary outcome variables. Response was defined as a 50\% decrease in the HDRS score at endpoint. Remission was defined as a HDRS score of 6 or less at endpoint.

In an 8-week dose-finding study involving 711 patients (18-65 years old), Loo and colleagues ${ }^{58}$ compared 3 doses of agomelatine (1, 5, and $25 \mathrm{mg} /$ day), placebo, and paroxetine $20 \mathrm{mg} /$ day (as an active comparison). Agomelatine $25 \mathrm{mg}$ (but not the 2 lower doses) and paroxetine were significantly more effective than placebo. The mean difference in HDRS was 2.57 for agomelatine $(25 \mathrm{mg})$ versus placebo $(P=0.034)$ and 2.25 for paroxetine versus placebo $(P=0.030)$. Response rates for agomelatine $1 \mathrm{mg}$ (62.5\%) and $25 \mathrm{mg}$ (61.5\%) were significantly better than for placebo (46.3\%), but agomelatine $5 \mathrm{mg}(51.4 \%)$ and paroxetine (56.3\%) were not significantly higher than placebo. Remission rates for agomelatine $25 \mathrm{mg}$ (30.4\%) and paroxetine (25.7\%) were significantly greater than for placebo (15.4\%), but the rates for agomelatine $1 \mathrm{mg}$ $(21.3 \%)$ and $5 \mathrm{mg}(17.8 \%)$ were not significantly better than placebo. The overall rate of treatment-emergent adverse events (TEAEs) was similar for agomelatine (51\%) and placebo (55\%), but slightly higher for paroxetine (66\%). Most adverse events were experienced as mild to moderate in severity. There was no significant difference in drop-out rates due to TEAEs between agomelatine, placebo, and paroxetine. Reported side effects of headache, anxiety, abdominal pain, diarrhea, nausea, somnolence, insomnia, rhinitis, and dry mouth were no different between agomelatine and placebo, 
Table I Summary of randomized controlled clinical efficacy studies of agomelatine

\begin{tabular}{|c|c|c|c|}
\hline Study (Reference) & Design & Comparison & Study outcome \\
\hline \multirow[t]{5}{*}{ Loo et $\mathrm{al}^{58}$} & Efficacy and safety & AGO I mg & $\mathrm{AGO} I \mathrm{mg}=\mathrm{PBO}$ \\
\hline & 8 weeks & AGO 5 mg & AGO 5 mg = PBO \\
\hline & 711 subjects & AGO $25 \mathrm{mg}$ & AGO $25 \mathrm{mg}>>$ PBO \\
\hline & & PBO & PAR $>>$ PBO \\
\hline & & PAR $20 \mathrm{mg}$ & \\
\hline \multirow[t]{3}{*}{ Kennedy and Emsley ${ }^{59}$} & Efficacy and safety & AGO $25-50 \mathrm{mg}$ & AGO $>>$ PBO \\
\hline & 6 weeks & PBO & \\
\hline & 212 subjects & & \\
\hline \multirow[t]{3}{*}{ Olie and Kasper ${ }^{60}$} & Efficacy and safety & AGO $25-50 \mathrm{mg}$ & $\mathrm{AGO}>>\mathrm{PBO}$ \\
\hline & 6 weeks & PBO & \\
\hline & 238 subjects & & \\
\hline \multirow[t]{3}{*}{ CL3-022 25} & Efficacy and safety & AGO $25 \mathrm{mg}$ & $\mathrm{AGO}=\mathrm{PBO}$ \\
\hline & 6 weeks & PBO & $\mathrm{FLX}>>\mathrm{PBO}$ \\
\hline & 419 subjects & $\mathrm{FLX} 20 \mathrm{mg}$ & \\
\hline \multirow[t]{3}{*}{ CL3-023 25} & Efficacy and safety & AGO $25 \mathrm{mg}$ & $\mathrm{AGO}=\mathrm{PBO}$ \\
\hline & 6 weeks & PBO & $\mathrm{PAR}=\mathrm{PBO}$ \\
\hline & 418 subjects & PAR 20 mg & \\
\hline \multirow[t]{4}{*}{ CL3-024 ${ }^{25}$} & Efficacy and safety & AGO $25 \mathrm{mg}$ & $\mathrm{AGO}=\mathrm{PBO}$ \\
\hline & 6 weeks & AGO $50 \mathrm{mg}$ & $\mathrm{FLX}=\mathrm{PBO}$ \\
\hline & 607 subjects & $\mathrm{PBO}$ & \\
\hline & & $\mathrm{FLX} 20 \mathrm{mg}$ & \\
\hline \multirow[t]{3}{*}{ CL3-02625 } & Efficacy and safety in elderly & AGO $25 \mathrm{mg}$ & $\mathrm{AGO}=\mathrm{PBO}$ \\
\hline & 6 weeks & PBO & \\
\hline & 218 subjects & & \\
\hline \multirow[t]{3}{*}{ Goodwin et $\mathrm{al}^{61}$} & Relapse prevention & AGO $25-50 \mathrm{mg}$ & AGO $>>$ PBO \\
\hline & 34 weeks & PBO & \\
\hline & 339 subjects & & \\
\hline \multirow[t]{3}{*}{ CL3-02I 25} & Relapse prevention & AGO $25 \mathrm{mg}$ & $\mathrm{AGO}=\mathrm{PBO}$ \\
\hline & 34 weeks & PBO & \\
\hline & 367 subjects & & \\
\hline
\end{tabular}

Notes: $>>$ denotes significantly better than; = denotes no significant difference.

Abbreviations: $\mathrm{AGO}$, agomelatine; $\mathrm{PBO}$, placebo; $\mathrm{PAR}$, paroxetine; $\mathrm{FLX}$, fluoxetine.

but nausea was significantly more common with paroxetine compared to placebo and agomelatine. There were no significant differences among the groups with respect to weight changes, cardiovascular effects, or laboratory studies.

In a second 6-week study involving 212 patients (18-65 years old), Kennedy and Emsley ${ }^{59}$ compared agomelatine 25 to $50 \mathrm{mg}$ /day and placebo. After an initial 2-week treatment with agomelatine $25 \mathrm{mg} /$ day or placebo, the study medication dosage of patients with poor response was increased under double-blind conditions to agomelatine $50 \mathrm{mg} /$ day or matching placebo. Among the 106 patients taking agomelatine, 69 patients stayed at $25 \mathrm{mg} /$ day and 36 patients increased to $50 \mathrm{mg} /$ day. Agomelatine (both doses pooled) was significantly more effective than placebo. The mean difference in HDRS was 2.30 for agomelatine versus placebo $(P=0.026)$. Response rates for agomelatine $(49.1 \%)$ and placebo (34.3\%) were significantly different, but remission rates for agomelatine (20.8\%) and placebo (13.3\%) were not significantly different. The overall rate of TEAEs was similar for agomelatine (30\%) and placebo (36\%). Most TEAEs were mild to moderate in severity. Drop-out rates due to TEAEs were similar for placebo (4.8\%) and agomelatine (4.7\%). Dizziness and rhinitis were more common with agomelatine compared to placebo, whereas headache, nausea, fatigue, dry mouth, and diarrhea were more common with placebo compared to agomelatine. There were no differences between agomelatine and placebo with respect to weight changes, cardiovascular effects, or laboratory studies.

In a third 6-week study involving 238 patients (18-65 years old), Olie and Kasper ${ }^{60}$ compared agomelatine 25 to $50 \mathrm{mg}$ /day and placebo. If patients did not respond adequately after 2 weeks of agomelatine $25 \mathrm{mg}$ /day or placebo, the dosage was increased under double-blind conditions to $50 \mathrm{mg}$ /day or matching placebo. Among the 118 patients on agomelatine, 82 stayed at $25 \mathrm{mg} /$ day and 29 increased to $50 \mathrm{mg}$ /day. Agomelatine (both doses pooled) was significantly more effective than placebo. The mean difference in HDRS was 3.18 for agomelatine versus placebo $(P=0.002)$. 
Response rates for agomelatine $(54.3 \%)$ and placebo (35.3\%) were significantly different, but remission rates for agomelatine (17.2\%) and placebo (11.8\%) were not significantly different. The overall rate of TEAEs was similar for agomelatine $(42 \%)$ and placebo $(43 \%)$. Most TEAEs were mildly to moderately severe. Drop-out rates due to TEAEs were slightly higher for placebo (5.8\%) compared to agomelatine (3.4\%). Fatigue, nausea, dizziness, rhinitis, and dry mouth were no different between agomelatine and placebo, but headache was significantly more common with placebo compared to agomelatine. Sexual functioning, weight changes, cardiovascular effects, and laboratory studies did not differ between groups.

In a 6-week unpublished study (study CL3-022) ${ }^{25}$ involving 419 patients (18-60 years old), the investigators compared agomelatine $25 \mathrm{mg} /$ day, placebo, and the SSRI drug fluoxetine $20 \mathrm{mg} /$ day (as an active comparison). Agomelatine was not significantly more effective than placebo (the mean difference in HDRS was 1.4 for agomelatine versus placebo). Response rates for agomelatine (53\%) and placebo (47\%) were not significantly different. Remission rates for agomelatine and placebo also were not significantly different (actual rates were not reported). By contrast, fluoxetine was significantly more effective than placebo (the mean difference in HDRS was 2.59 for fluoxetine versus placebo). Response and remission rates for fluoxetine were not reported. There was no significant difference in drop-out rates due to TEAEs during the 6-week trial between agomelatine, placebo, and fluoxetine. At the end of 6 weeks, subjects responding to agomelatine or to fluoxetine were eligible for an 18-week double-blind placebo-controlled extension phase. Among these subjects, the final depression score was lower in the agomelatine group than in the placebo group, but the difference was not statistically significant. Nine subjects in the agomelatine group (14.3\%), 20 in the placebo group (33.3\%), and 13 in the fluoxetine group (17.8\%) relapsed during the continuation phase. The survival curve time to relapse analysis showed a statistically significant difference favoring active drug (agomelatine or fluoxetine) compared to placebo.

In another 6-week unpublished study (study CL3-023) ${ }^{25}$ involving 418 patients (18-60 years old), the investigators compared agomelatine $25 \mathrm{mg}$ /day, placebo, and paroxetine $20 \mathrm{mg} /$ day (as an active comparison). Agomelatine was not significantly more effective than placebo (the mean difference in HDRS was 0.8 for agomelatine versus placebo). Response and remission rates for agomelatine and placebo were not significantly different (actual rates were not reported).
Paroxetine was not significantly more effective than placebo (the mean difference in HDRS was 1.6 for paroxetine versus placebo). Response and remission rates for paroxetine were not reported. During the acute 6-week trial, there was no significant difference in drop-out rates due to TEAEs between agomelatine, placebo, and paroxetine. At the end of 6 weeks, subjects responding to agomelatine or to paroxetine were eligible for an 18-week, double-blind, placebo-controlled extension phase. There were no significant differences in outcome between active drugs or placebo in the extension phase. Drop-out rates due to TEAEs during the extension phase were similar for agomelatine (3.3\%) and placebo (3.4\%), but higher for paroxetine (8.6\%).

In the last 6-week unpublished study (study CL3-024) 25 $^{25}$ involving 607 patients (18-65 years old), the investigators compared two doses of agomelatine $(25 \mathrm{mg} /$ day and $50 \mathrm{mg} /$ day), placebo, and fluoxetine $20 \mathrm{mg} /$ day (as an active comparison). Agomelatine $25 \mathrm{mg}$ was not significantly more effective than placebo (the mean difference in HDRS was 1.4 for agomelatine versus placebo). Agomelatine $50 \mathrm{mg}$ also was not significantly more effective than placebo (HDRS data were not reported). Similarly, fluoxetine was not significantly more effective than placebo (the mean difference in HDRS was 0.53 for fluoxetine versus placebo). Response and remission rates for agomelatine and for fluoxetine were not reported. At the end of 6 weeks, subjects responding to agomelatine or to fluoxetine were eligible for an 18-week, double-blind, placebo-controlled extension phase. In this phase, there were no significant differences in outcome between active drugs or placebo. Throughout the acute and extension phases of the study, there was no significant difference in drop-out rates due to TEAEs between agomelatine, placebo, and fluoxetine.

In addition to the six pivotal studies, a 6-week unpublished study (study CL3-026) ${ }^{25}$ involving 218 elderly patients (60 years and older) was conducted comparing agomelatine $25 \mathrm{mg} /$ day and placebo (see Table 1). For this study, the Montgomery Asberg Depression Rating Scale (MADRS) was the primary outcome measure. The primary outcome efficacy variable was the MADRS score for agomelatine versus placebo at endpoint. The response rate based on the MADRS was considered a secondary outcome variable. Response was defined as a $50 \%$ decrease in the MADRS score at endpoint. Remission rates were not reported in this study. Agomelatine was not significantly more effective than placebo (the mean difference in MADRS was 0.19 for agomelatine versus placebo). Response rates for agomelatine (46\%) and placebo $(52 \%)$ were not significantly different. At the end of 6 weeks, 
subjects responding to acute treatment were eligible for an 18-week, double-blind, placebo-controlled extension phase. There were no significant differences in outcome between active drugs or placebo during the extension phase. In an unplanned post-hoc subgroup analysis of data from this study, agomelatine was significantly more effective than placebo among the subgroup of 86 patients with more severe levels of depression. In a post-hoc pooled analysis of data from the 3 published short-term pivotal studies,${ }^{58-60}$ which included subjects over 60 years of age, a significant antidepressant effect was observed in the subgroup of 53 patients who were 60-66 years old.

The efficacy of agomelatine for children and adolescents (younger than 18 years) having major depression has not been investigated.

\section{Longer-term relapse prevention studies of agomelatine for major depression}

The effectiveness of agomelatine has been investigated in 1 published and 1 unpublished longer-term, randomized, double-blind, placebo-controlled relapse prevention study in patients with recurrent major depression (see Table 1). In both studies, subjects were eligible for the randomized phase if they had a response (50\% reduction in HDRS) or a remission (HDRS less than seven) after acute open-label treatment with agomelatine. In both studies, relapse was defined as one of the following: a HDRS score greater than 15, withdrawal for lack of efficacy, suicide, or suicide attempt.

In a trial reported by Goodwin and colleagues, ${ }^{61}$ 492 patients (19-65 years old) were initially treated openly with agomelatine 25 to $50 \mathrm{mg}$ /day for up to 10 weeks. After 2 weeks of agomelatine $25 \mathrm{mg}$ /day, the dosage for poor responders was increased to $50 \mathrm{mg}$ /day. The 339 patients who were responders or remitters were then randomized to receive double-blind treatment with agomelatine (at their current dose) or placebo for up to 24 weeks until they suffered a relapse. At the time of randomization, $22 \%$ of subjects were taking $50 \mathrm{mg} /$ day and the remaining patients were taking $25 \mathrm{mg} /$ day. Among all patients, 70\% taking agomelatine completed the 24-week study compared to only $52 \%$ taking placebo. Overall, agomelatine patients had a significantly lower cumulative relapse rate (22\%) compared to placebo patients $(47 \%)$. In a post-hoc data analysis, the cumulative relapse rate for agomelatine-treated patients $(22 \%)$ was significantly lower than the rate for placebotreated patients (45\%) among the subgroup of patients with more severe levels of depression. The overall rate of TEAEs was similar for agomelatine (56\%) and placebo (56\%). The most common TEAEs were headache (agomelatine $10.3 \%$; placebo $7.5 \%$ ), rhinitis (agomelatine 6.7\%; placebo 9.8\%), and back pain (agomelatine 6.1\%; placebo 3.4\%). Agomelatine was not associated with significant effects on sexual functioning, weight, cardiovascular effects, or laboratory studies.

In the unpublished trial (study CL3-021), ${ }^{25} 551$ patients (19-67 years old) were initially treated openly with agomelatine $25 \mathrm{mg}$ /day for up to 8 weeks. The 367 patients who were responders or remitters were then randomized to receive double-blind treatment with agomelatine or placebo for up to 34 weeks until they suffered a relapse. Overall, agomelatine-treated patients had a similar cumulative relapse rate $(26 \%)$ compared to placebo-treated patients (24\%). In a post-hoc data analysis, the cumulative relapse rate for agomelatine-treated patients $(21 \%)$ was significantly lower than the rate for placebo-treated patients (31\%) among the subgroup of patients with more severe levels of depression.

\section{Tolerability and safety of agomelatine in placebo- controlled pivotal trials}

The tolerability and safety of agomelatine has been assessed extensively in the six short-term pivotal studies. ${ }^{25}$ These studies included 1120 patients taking agomelatine 25 to $50 \mathrm{mg} /$ day, 998 patients taking placebo, 284 patients taking fluoxetine $20 \mathrm{mg} / \mathrm{day}$, and 283 patients taking paroxetine $20 \mathrm{mg} /$ day. The overall rate of TEAEs was similar for agomelatine (53\%), placebo (52\%), and fluoxetine (49\%), but slightly higher for paroxetine (68\%). The most commonly reported adverse events for agomelatine (in descending order of their incidence) were headache, nausea, dizziness, dry mouth, diarrhea, somnolence, fatigue, upper abdominal pain, and anxiety. Each of these adverse events was reported in less than $15 \%$ of patients. The incidence of adverse events was slightly higher for the $50 \mathrm{mg}$ dose compared to the $25 \mathrm{mg}$ dose. Most agomelatine adverse events were mildly to moderately severe. The only adverse events significantly higher for agomelatine compared to placebo were dizziness, paresthesias, and blurred vision. The most commonly reported adverse events for the two SSRI drugs (in descending order of their incidence) were nausea, headache, dry mouth, diarrhea, somnolence, fatigue, insomnia, dizziness, and anxiety. Each of these was reported in less than $16 \%$ of patients. The largest 
difference between agomelatine and the SSRI drugs in the incidence of any adverse event was for nausea, which favored agomelatine.

In addition to the two relapse prevention studies, many of the short-term pivotal studies had optional extension phases. Based on data collected from the relapse prevention and extension phase studies altogether, longer-term tolerability and safety of agomelatine has been assessed for up to 24 weeks. ${ }^{25}$ These data include 511 patients taking agomelatine 25 to $50 \mathrm{mg} /$ day, 406 patients taking placebo, 222 patients taking fluoxetine $20 \mathrm{mg} /$ day, and 105 patients taking paroxetine $20 \mathrm{mg}$ /day. The overall rate of TEAEs was similar for agomelatine (39\%) and placebo (38\%), but slightly lower for fluoxetine (32\%) and slightly higher for paroxetine $(45 \%)$. The rate of TEAEs was higher for agomelatine $50 \mathrm{mg} /$ day (48\%) than for $25 \mathrm{mg} /$ day $(36 \%)$. The most commonly reported adverse events for agomelatine (in descending order of their incidence) were headache, back pain, and insomnia, each of which was reported in less than $10 \%$ of patients. The only adverse event that was significantly higher for agomelatine compared to placebo was insomnia. The most commonly reported adverse events for the 2 SSRI drugs (in descending order of their incidence) were headache, diarrhea, insomnia, and anxiety. Each of these was reported in less than $9 \%$ of patients. There were no major differences in long-term tolerability between the SSRI drugs and agomelatine.

A limited number of patients (400) took agomelatine for 1 year. $^{25}$ There were few TEAEs during this extended time (each occurring in fewer than $3 \%$ of patients), and none were different than what was seen in the short-term and long-term studies. No comparisons were available with placebo or SSRI drugs.

Among all patients enrolled in clinical studies, there was no significant difference in the overall rate of serious adverse events for agomelatine $25 \mathrm{mg} /$ day (4.1\%) and $50 \mathrm{mg} /$ day (4.4\%), compared to placebo (4.1\%). The most common serious adverse events were suicide attempts (agomelatine $0.6 \%$ versus placebo $0.4 \%$ ), depression (agomelatine $0.5 \%$ versus placebo $0.8 \%$ ), and falls (agomelatine $0.3 \%$ versus placebo $0.3 \%$ ). In the depression trials, deaths (all but 1 due to suicide) were reported in four of 3956 patients taking agomelatine $(0.1 \%), 1$ of 826 patients taking placebo $(0.1 \%)$, and three of 449 patients taking paroxetine $(0.7 \%)$. In nondepression clinical trials, the percentage of deaths among patients taking agomelatine (16 of 782 patients; 2\%) was higher than for patients taking placebo (one of 327 patients; 0.3\%). Fifteen of the 16 deaths in agomelatine-treated patients occurred in a study of 356 elderly patients who had Alzheimer's dementia (a mortality rate of $4.2 \%$ ).

In the clinical trials, significant elevations of liver enzymes (ie, increases greater than three times the upper limit of normal) occurred in $1.39 \%$ of patients taking agomelatine $50 \mathrm{mg} /$ day, $1.04 \%$ taking $25 \mathrm{mg}$ /day, and $0.72 \%$ taking placebo. ${ }^{25}$ These liver reactions occurred at various times throughout the 6-month observation period of these studies. They were detected in patients only through laboratory monitoring, because they did not have obvious clinical signs or symptoms indicating liver injury. Some reactions recovered during continued treatment and some recovered after treatment discontinuation. Serious liver reactions, including hepatitis and enzyme elevations greater than ten times the upper limit of normal, were reported less frequently. One patient developed hepatitis that did not recover at follow-up (2.5 years after discontinuation of agomelatine). These reactions in humans are not inconsistent with the findings from animal studies. Repeated dose toxicity studies in rats and monkeys have indicated that the liver is the target organ of toxicity. Agomelatine causes hepatic enzyme induction in these animals, and they consequently showed enlarged livers or hepatocellular hypertrophy.

As described in the section on pharmacokinetics, liver insufficiency results in a significant increase in the exposure to agomelatine. Because the safety of such large concentrations of agomelatine is unknown, it should not be used in patients with hepatic insufficiency, such as cirrhosis or other active liver disease.

Agomelatine does not significantly affect renal function. In patients with normal hepatic function, impaired renal function would be expected to result in greater exposure to agomelatine metabolites rather than to parent drug. The available safety data from the clinical trials did not demonstrate any significant tolerability or safety issues with the use of agomelatine compared to placebo among patients with mildly to moderately impaired renal function. However, experience in patients with more severe renal impairment is unknown. Although agomelatine can be used in patients with renal impairment, such patients should be monitored more closely.

Agomelatine has not been associated with significant weight gain or adverse metabolic effects. Only 4 of 400 patients taking it for one year gained weight. It does not have adverse cardiac effects (eg, electrocardiogram or blood pressure changes). With the exception of potential adverse liver effects, animal and human studies have not identified significant toxicity, even with excessively high doses. 
Among patients older than 65 years, safety data are available on 109 patients taking agomelatine and 76 taking placebo. ${ }^{25}$ Rates of TEAEs were similar for agomelatine (63.3\%) and placebo (59.2\%). Discontinuation rates due to TEAEs were $12.8 \%$ for agomelatine and $9.2 \%$ for placebo. Serious adverse events occurred in $4.6 \%$ of patients taking agomelatine and in $5.2 \%$ taking placebo.

\section{Tolerability and safety of agomelatine in nonpivotal clinical trials}

In addition to the randomized placebo-controlled pivotal studies, data on the tolerability and efficacy of agomelatine have been investigated in several other clinical trials.

In a 12-week randomized double-blind study involving 276 male and female patients with depression (18-60 years old), Kennedy and colleagues ${ }^{62}$ compared agomelatine $(50 \mathrm{mg} /$ day $)$ and the serotonin-norepinephrine reupake inhibitor (SNRI) antidepressant venlafaxine (titrated from $75 \mathrm{mg} /$ day to $150 \mathrm{mg} /$ day after 2 weeks). The primary objective of this study was to compare the sexual effects of these 2 drugs using data taken from the Sex Effects Scale. On the primary outcome measure of sexual function, there was a numerical advantage favoring agomelatine over venlafaxine, but these results were not statistically significant. Only several of the secondary outcome measures of sexual function showed statistically significant differences in favor of agomelatine. The overall rate of TEAEs for agomelatine (20\%) was lower than for venlafaxine $(38 \%)$. The most commonly reported adverse events were nausea (agomelatine $11.7 \%$ versus venlafaxine $17.3 \%$ ) and headache (agomelatine $10.2 \%$ versus venlafaxine $7.9 \%$ ). Discontinuation rates due to TEAEs were $2.2 \%$ for agomelatine and $8.6 \%$ for venlafaxine. There was no difference in antidepressant efficacy between the two drugs.

The objective of another study was to compare the sexual effects of agomelatine and paroxetine in healthy nondepressed male subjects. ${ }^{63}$ In this 8 -week double-blind study, 92 subjects (18-30 years old) were randomized to 1 of 2 doses of agomelatine ( 25 or $50 \mathrm{mg} /$ day), placebo, or paroxetine $(20 \mathrm{mg} /$ day $)$. On the primary and secondary outcome measures of sexual function (using data taken from the Psychotropic-Related Sexual Dysfunction Questionnaire), agomelatine (at both doses) and placebo were not significantly different. These three groups were significantly less impaired than the paroxetine-treated group.

In a 6-week randomized double-blind study involving 332 male and female depressed patients (18-65 years old), Lemoine and colleagues ${ }^{64}$ compared agomelatine
(25-50 mg/day) and venlafaxine (75-150 mg/day). The primary objective of this study was to compare the sleep effects of these two drugs using the Leeds Sleep Evaluation Questionnaire. On the primary outcome measure of sleep, and on most of the secondary outcome measures, there was a clinically modest, but statistically significant advantage favoring agomelatine over venlafaxine. The overall rate of TEAEs was slightly lower for agomelatine (52\%) compared to venlafaxine (57\%). The most commonly reported adverse events (agomelatine versus venlafaxine, respectively) were nausea ( $6.0 \%$ versus $22.6 \%$ ), headache (9.6\% versus $11.9 \%)$, dizziness ( $1.8 \%$ versus $9.5 \%)$, vomiting $(1.2 \%$ versus $4.8 \%)$, diarrhea ( $4.8 \%$ versus $1.8 \%)$, and somnolence (3.6\% versus $4.8 \%$ ). Rates of discontinuation due to TEAEs were $4.2 \%$ for agomelatine and $13.2 \%$ for venlafaxine. There was no difference in antidepressant efficacy between the 2 drugs.

Another 6-week randomized double-blind study whose primary objective was to compare agomelatine ( $25-50 \mathrm{mg} /$ day) and the SSRI drug sertraline $(50-100 \mathrm{mg} /$ day) on the rest-activity cycle of depressed patients has been completed. Neither the results based on the primary outcome measure nor other tolerability and safety data have been reported. ${ }^{65}$ As a pre-specified secondary outcome, antidepressant efficacy was assessed. Although complete details of this study have not been published, ${ }^{66}$ the data submitted to the European Medicines Agency demonstrated a small statistically significant treatment effect of 1.68 on the HDRS in favor of agomelatine over sertraline..$^{25}$

The effects of abruptly discontinuing agomelatine and paroxetine have been compared in a randomized, double-blind, placebo-controlled study ${ }^{67}$ After 12 weeks of double-blind treatment with agomelatine $25 \mathrm{mg}$ /day or paroxetine $20 \mathrm{mg}$ /day, 192 depressed patients who were in sustained remission were randomized to continue taking their current drug or to switch to placebo for 2 weeks. Discontinuation symptoms (rated on the Discontinuation Emergent Signs and Symptoms checklist) were significantly higher in the paroxetine-discontinuation group during the first week of placebo, but not during the second week. Discontinuation symptoms were not observed in the agomelatine-discontinuation group during the first or second week of placebo.

In a 12-week randomized, double-blind, placebocontrolled study involving 121 male and female patients with generalized anxiety disorder (18-65 years old), Stein

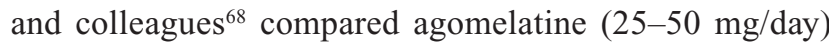
and placebo. If patients did not respond adequately after 
2 weeks of agomelatine $25 \mathrm{mg} /$ day or placebo, the dosage was increased under double-blind conditions to $50 \mathrm{mg} /$ day or matching placebo. Agomelatine was significantly more effective than placebo on the primary outcome measure of anxiety (total score on the Hamilton Anxiety Rating Scale). The overall rate of TEAEs was similar for agomelatine $25 \mathrm{mg}$ (36.1\%), $50 \mathrm{mg}$ (38.5\%), and placebo (34.5\%). The most common TEAEs that were reported more frequently in the agomelatine than in the placebo groups were dizziness $(7.9 \%$ versus $3.4 \%$ ) and nausea (4.8\% versus $1.7 \%$ ). Most TEAEs were mildly to moderately severe. Drop-out rates due to TEAEs were slightly lower for placebo (none) compared to agomelatine (1.6\%). Cardiovascular effects and laboratory studies did not differ between groups.

\section{Tolerability and safety of agomelatine in overdoses, pregnancy, and pediatric populations}

There is limited clinical experience with agomelatine overdose in humans. Reported overdoses with agomelatine (up to $525 \mathrm{mg}$ ) have not resulted in significant or serious sequelae. It should also be noted that in the earliest clinical studies, healthy subjects took agomelatine doses as high as $1200 \mathrm{mg}$ and the maximum tolerated dose was $800 \mathrm{mg}$. In animals, the LD50 dose is at least 100 times greater than the comparable human dose. Hence, the acute toxic effects of agomelatine have a relatively favorable safety profile.

There are no specific data on the safety of agomelatine during pregnancy or with breast feeding. Two phase I studies ( 1 in men, 1 in women) did not demonstrate any adverse effect of agomelatine on various gonadotrophic hormones, spermogram, or menstrual cycle. Reproduction toxicity studies in animals did not reveal any adverse effect of agomelatine on fertility or on embryonal or fetal development.

A small open-label sleep study in 9 pediatric patients (6-17 years old) with Smith-Magenis syndrome reported that agomelatine was well tolerated ${ }^{69}$ The tolerability and safety of agomelatine has not otherwise been studied in children and adolescents.

\section{Clinical considerations on the use of agomelatine and areas for further study}

The antidepressant efficacy of agomelatine has been systematically assessed in 6 short-term acute studies and
2 longer-term relapse prevention studies. Three acute trials demonstrated clinically modest, but statistically significant benefits over placebo. Three acute trials did not find agomelatine to be more effective than placebo. Low-dose fluoxetine was more effective than placebo in one of these negative trials. Low-dose fluoxetine and low-dose paroxetine were not more effective than placebo in the other 2 negative trials. The lack of efficacy of the active comparator drugs in these 2 trials was believed mainly to be due to an insufficient dose and possibly to unusually high placebo responder rates. ${ }^{25}$

The only controlled study conducted in elderly patients did not demonstrate a significant benefit for agomelatine compared to placebo. Agomelatine was more effective than placebo in only 1 of the 2 relapse prevention studies. A meta-analysis of the 6 acute trials submitted to the European Medicines Agency demonstrated a small statistically significant treatment effect of about 1.5 on the HDRS in favor of agomelatine over placebo. ${ }^{25}$ The results from these studies suggest that agomelatine $25 \mathrm{mg}$ /day is probably less effective than other antidepressant drugs. In their summary in approving agomelatine, the European Medicines Agency concluded that some positive treatment effect of agomelatine in major depression was demonstrated, but that the magnitude of effect was considered to be of marginal clinical relevance. ${ }^{25}$

In these clinical trials, agomelatine was generally well tolerated compared to placebo. The most common side effects associated with agomelatine are headache, nausea, dizziness, dry mouth, diarrhea, somnolence, fatigue, upper abdominal pain, and anxiety. The relatively benign side effect profile of agomelatine (especially the lack of clinically significant weight gain, the low risk of sexual dysfunction, the low incidence of gastrointestinal symptoms, and the absence of discontinuation symptoms) is different than and compares favorably to SSRI and SNRI drugs. However, the overall tolerability of agomelatine in head-to-head comparisons was not substantially better than active drug comparitors, as evidenced by the roughly similar rates of discontinuation due to TEAEs.

One particular concern with the use of agomelatine is liver function. Significant elevations of liver enzymes are common. These were sometimes very serious and included rare cases of hepatitis. Because these hepatic reactions were not predictable based on clinical symptoms or the duration of treatment, monitoring of liver enzyme levels of all patients has been recommended before starting treatment, after 6,12 , and 24 weeks of treatment, and then thereafter when clinically indicated based on the judgment of the treating physician. 
"Real world" patients in general practice are likely to be quite different than the patients enrolled in clinical trials. ${ }^{70}$ As a result, the relative safety of agomelatine is unknown when used in patients who might have undetected liver impairment or liver disease or in patients who are at risk for developing liver disease. Commonly encountered clinical scenarios, for example, would include treating depressed patients at risk for developing viral hepatitis and treating patients who use alcohol, acetaminophen, or other prescription and nonprescription drugs that affect the liver. Also, pharmacokinetic studies indicate that even mild hepatic insufficiency results in very elevated concentrations of agomelatine. For this reason, agomelatine is contraindicated in patients with any degree of liver impairment, such as cirrhosis or other active liver disease. All of these issues regarding the liver are of obvious concern for the routine use of agomelatine. The liver precautions and the need for laboratory monitoring are a distinct disadvantage for the use of agomelatine compared to many other antidepressant drugs.

Agomelatine is metabolized primarily by CYP-1A2 enzyme in the liver, with lesser metabolic contributions from CYP-2C9/2C19. Based on this, the use of agomelatine is contraindicated in patients taking drugs strongly inhibiting this enzyme (eg, fluvoxamine). Moderate inhibitors of CYP-1A2, such as estrogen-containing oral contraceptives and paroxetine, increase agomelatine concentrations to a lesser degree. Patients taking any drugs having moderate inhibitory effects on CYP-1A2 or drugs having inhibitory effects on CYP-2C9/2C19 should simply be monitored for increased adverse effects. It is possible that patients taking one or more drugs that have moderate metabolic enzyme inhibiting effects would benefit from taking and staying on lower agomelatine doses (ie, $25 \mathrm{mg} /$ day). By contrast, patients who smoke and those taking drugs that induce CYP-1A2 enzyme activity (eg, the proton pump inhibitor drug omeprazole) may be more likely to require doses of $50 \mathrm{mg}$ /day or possibly even higher doses (although this has not been evaluated clinically).

In patients with normal hepatic function, impaired renal function may result in higher concentrations of agomelatine metabolites rather than the parent drug. Patients with renal impairment do not require any special laboratory monitoring with the use of agomelatine, but they should still be monitored for increased adverse effects.

The treatment of depression is provided in 3 phases, and each phase has different therapeutic goals. ${ }^{71}$ The acute phase, which typically lasts 6 to 12 weeks, refers to the initial treatment period, where the treatment is administered with the goal of achieving full symptom remission. The continuation phase, typically 4 to 9 months, follows acute phase treatment and is recommended for all patients. The goal of continuation phase treatment is to prevent early relapse and to allow further symptomatic and psychosocial functional improvement. The goal of maintenance phase treatment is to prevent further recurrences of depression among patients who have a high risk of developing depression again. Such risk factors include three or more previous episodes of major depression, chronic depression, residual or persistent depressive symptoms despite adequate treatment, and severe or disabling episodes of depression. The length of maintenance treatment will depend on the number of risk factors, and may range from one year or more or even to life-long treatment. Compared to other antidepressant drugs, agomelatine has not been systematically studied beyond continuation phase treatment.

Given the availability of a wide variety of potentially effective therapies, how does one choose among these treatments? Because of the expected delay in onset of antidepressant effect, adherence to treatment is very important. Adherence also is obviously important for longer-term continuation and maintenance treatment. The initial selection of medication, as well as adherence to treatment, are influenced by such factors as cost and availability, patient preference, past treatment history, family treatment history, clinical symptoms, expected side effect profile and safety, and the need for medical/laboratory monitoring. ${ }^{72}$ Comparative data on the relative efficacy, tolerability, safety, and acceptability of the antidepressant drug treatment options would be helpful, but is often lacking. ${ }^{73,74}$

As a new product, relatively less is known about many of these issues with agomelatine vis-à-vis other antidepressant drugs. Given the current status of agomelatine, what areas deserve further study? Because the oral bioavailability of agomelatine is very low at recommended doses, there is a critical need to investigate its pharmacokinetics, efficacy, tolerability, and safety in patients taking doses higher than $50 \mathrm{mg} /$ day. In an early 4-week phase II safety and efficacy pilot study, 28 hospitalized depressed patients (18-65 years old) were randomized to receive double-blind treatment at one of two doses of agomelatine ( $5 \mathrm{mg} /$ day versus $100 \mathrm{mg} /$ day). ${ }^{25,75}$ There was no placebo control and the MADRS was the primary outcome measure. MADRS scores decreased significantly in both groups and there was no significant difference between groups. One subject in each group dropped out due to adverse events and two dropped out in each group due to lack of efficacy. Acceptability of both doses was reported to be good, but there were slightly more TEAEs and severe 
TEAEs in the $100 \mathrm{mg}$ group. There were no observed adverse cardiovascular effects or abnormal laboratory studies in either group. Future clinical studies should investigate the efficacy, tolerability, and safety of agomelatine in the 50 to $100 \mathrm{mg}$ /day dose range.

The efficacy, tolerability, and safety of agomelatine also should be further investigated in "real world" patient populations, in pediatric and in geriatric patients, and in maintenance therapy studies extending for more than 6 months. Information is needed about the effects of agomelatine during pregnancy and breast feeding. ${ }^{76,77}$ The relative effectiveness of agomelatine also should be evaluated in comparison with SSRIs, SNRIs, and other antidepressant drugs using their full dose range, rather than minimally effective doses. ${ }^{78}$ Given agomelatine's pharmacology and clinical profile, comparisons with the antidepressant drugs bupropion, nefazodone, and mirtazapine would be of particular interest. Bupropion is a nonserotonergic drug that has relatively benign sexual, gastrointestinal, and weight effects. Nefazodone is a 5HT2A receptor antagonist, with favorable sleep, anxiety, sexual, gastrointestinal, and weight effects. Mirtazapine has a complicated pharmacology that includes 5HT2A, 5HT2C, and 5HT3 receptor antagonism, and it has favorable anxiety, sleep, gastrointestinal, and sexual effects, but it is prone to sedation and weight gain. Trazodone also has 5HT2A and 5HT2C receptor antagonist effects. However, it is most commonly used in low doses as a hypnotic, and is rarely used as an antidepressant because of sedation and the risk of priapism (in men) when used at higher antidepressant therapeutic doses. The SSRI and SNRI drugs have been extensively investigated, approved, and marketed for the treatment of various anxiety disorders. Except for the recently published study of generalized anxiety disorder, agomelatine has not been investigated for the treatment of various anxiety disorders. ${ }^{68}$

The combined action at MT1, MT2, and 5HT-2C receptors, which may resynchronize disturbed circadian rhythms and abnormal sleep patterns, suggest that agomelatine might be particularly effective for the treatment of seasonal affective disorder as well as bipolar depression..$^{79,80}$ Preliminary open-label studies in these patient populations have suggested some benefit ${ }^{81,82}$ and further studies in these patient populations is clearly warranted.

For patients not responding adequately to an initial antidepressant medication, or for patients who cannot tolerate the medication, the two main treatment approaches are switching or augmentation. ${ }^{83}$ The unique pharmacology of agomelatine suggests a potential role not only as an appropriate switch agent for medication intolerance or nonresponse, but also as an augmentation agent that could be used in combination with other antidepressant drugs. Combining antidepressant drugs for treatment nonresponders is commonly done in clinical practice. One rationale for this strategy is that antidepressant drugs from different classes have distinct pharmacological properties. These distinct properties may complement each other when used in combination, potentially resulting in additive or synergistic neurobiological and clinical effects. For example, mirtazapine and some second generation antipsychotic drugs have each been shown to be effective as add-on therapies for treatment resistant depression. ${ }^{38,84}$ Based on the same rationale, combining agomelatine's particular pharmacology with other antidepressant drugs, especially SSRI and SNRI drugs, could be done to achieve a synergistic antidepressant effect. Similarly, the 5HT2C receptor antagonist effects of agomelatine might justify its use in combination with SSRI or SNRI drugs as a way to counter their serotonin-related adverse effects, such as sexual dysfunction, gastrointestinal complaints, or insomnia. Controlled studies investigating the use of agomelatine as a switch agent or in combination with other antidepressant drugs for these clinical purposes are warranted.

With the advent of newer generation antidepressant drugs such as agomelatine, are patients with depression better off than they were 10 years ago? Depression can be a devastating and sometimes difficult-to-treat illness, and it is always good to have alternative treatment options. The choice of drug treatment for an individual patient should be based as much as possible on the best unbiased clinical and scientific information available. Agomelatine does not have clinically significant advantages compared to other antidepressant drugs, and it has certain limitations and disadvantages. Because of its unique pharmacology and relatively benign tolerability profile, however, it may be a useful alternative for patients who do not respond to or cannot tolerate other antidepressant drugs.

\section{Disclosures}

Novartis (Past Grant Support).

\section{References}

1. Rush AJ, Kraemer HC, Sackeim HA, et al. Report by the ACNP task force on response and remission in major depression. Neuropsychopharmacol. 2006;31:1841-1853.

2. Howland RH. Chronic depression. Hosp Community Psychiatry. 1993;44:633-639.

3. Crown WH, Finkelstein S, Berndt ER, et al. The impact of treatmentresistant depression on health care utilization and costs. J Clin Psychiatry. 2002;63:963-971. 
4. Dunner DL, Rush AJ, Russell JM, et al. Prospective long-term multicenter study of the naturalistic outcomes of patients with treatment-resistant depression. J Clin Psychiatry. 2006;67:688-695.

5. Howland RH. Health status, health care utilization, and medical comorbidity in dysthymia. Int J Psychiatry Med. 1993;23:211-238.

6. Howland RH, Schettler PJ, Rapaport MH, et al. Clinical features and functioning of patients with minor depression. Psychother Psychosom. 2008;77:384-389.

7. Koplan C, Charuvastra A, Compton MT, et al. Prevention psychiatry. Psychiatr Ann. 2007;37:319-328.

8. Baune BT, Adrian I, Jacobi F. Medical disorders affect health outcome and general functioning depending on comorbid major depression in the general population. J Psychosomatic Res. 2007;62:109-118.

9. Carney RM, Freedland KE. Treatment-resistant depression and mortality after acute coronary syndrome. Am J Psychiatry. 2009;166:410-417.

10. Rivelli S, Jiang W. Depression and ischemic heart disease: What have we learned from clinical trials? Curr Opin Cardiol. 2007;22:286-291.

11. Carnethon MR, Biggs ML, Barzilay JI, et al. Longitudinal association between depressive symptoms and incident type 2 diabetes mellitus in older adults. Arch Intern Med. 2007;167:802-807.

12. Diem SJ, Blackwell TL, Stone KL, et al. Depressive symptoms and rates of bone loss at the hip in older women. J Am Geriactr Soc 2007;55:824-831.

13. Spernak SM, Moore PJ, Hamm LF. Depression, constructive thinking, and patient satisfaction in cardiac treatment adherence. Psychol Health Med. 2007;12:172-189.

14. Kilbourne AM, Reynolds CF, Good CB, Sereika SM, Justice AC, Fine MJ. How does depression influence diabetes medication adherence in older patients? Am J Geriat Psychiatry. 2005;13:202-210.

15. Mohr DC, Goodkin DE, Likosky W, Gatto N. Treatment of depression improves adherence to interferon beta-1b therapy for multiple sclerosis. Arch Neurology. 1997;54:531-533.

16. Safren SA, Hendricksen ES, Mayer KH, Mimiaga MJ, Pickard R, Otto MW. Cognitive-behavioral therapy for HIV medication adherence and depression. Cogn Behav Pract. 2004;11:415-424.

17. Knol MJ, Twisk JW, Beekman AT, Heine RJ, Snoek FJ, Pouwer F. Depression as a risk factor for the onset of type 2 diabetes mellitus A meta-analysis. Diabetologia. 2006;49:837-845

18. Gold DT, Solimeo S. Osteoporosis and depression: A historical perspective. Curr Osteoporos Rep. 2006;4:134-139.

19. Ilias I, Alesci S, Gold PW, Chrousos GP. Depression and osteoporosis in men: Association or casual link? Hormones. 2006;5:9-16.

20. O’Keane V, Marsh MS. Depression during pregnancy. BMJ. 2007;334:1003-1005.

21. Wisner KL, Zarin DA, Holmboe ES, et al. Risk-benefit decision making for treatment of depression during pregnancy. JAMA. 2000;157:1933-1940.

22. Rush AJ. Limitations in efficacy of antidepressant monotherapy. J Clin Psychiatry. 2007;68(Suppl 10):8-10.

23. Hamon M, Bourgoin S. Pharmacological profile of antidepressants: A likely basis for their efficacy and side effects? Eur Neuropsychopharmacol. 2006;16:S625-S632.

24. Dubocovich ML. Drug evaluation: Agomelatine targets a range of major depressive disorder symptoms. Curr Opin Investig Drugs. 2006;7: 670-680.

25. European Medicines Agency. CMHP Assessment Report For Valdoxan. URL: http://www.emea.europa.eu/humandocs/PDFs/EPAR/valdoxan/ H-915-en6.pdf. Accessed November 20, 2008

26. European Medicines Agency. Committee For Medicinal Products For Human Use Summary of Positive Opinion for Valdoxan. URL: http:// www.emea.europa.eu/pdfs/human/opinion/Valdoxan_57541108en.pdf. Accessed November 20, 2008

27. European Medicines Agency. Valdoxan Product Information. Summary of Product Characteristics. URL: http://www.emea.europa. eu/humandocs/PDFs/EPAR/valdoxan/H-915-PI-en.pdf.

28. US National Institutes of Health. URL: http://www.clinicaltrials.gov.
29. Zlotos DP. Recent advances in melatonin receptor ligands. Arch Pharm Chem Life Sci. 2005;338:229-247.

30. Wirz-Justice A. Biological rhythm disturbances in mood disorders. Int Clin Psychopharmacol. 2006;21(Suppl 1):S11-S15.

31. Wehr TA. Photoperiodism in humans and other primates: Evidence and implications. J Biol Rhythms. 2001;16:348-364.

32. Pandi-Perumal SR, Srinivasan V, Maestroni GJM, Cardinali DP, Poeggeler B, Hardeland R. Melatonin: Nature's most versatile biological signal? FEBS J. 2006;273:2813-2838.

33. Pandi-Perumal SR, Srinivasan V, Spence DW, Cardinali DP. Role of the melatonin system in the control of sleep: Therapeutic implications. CNS Drugs. 2007;21:995-1018.

34. Rajaratnam SMW, Cohen DA, Rogers NL. Melatonin and melatonin analogues. Sleep Med Clin. 2009;4:179-193.

35. Landolt HP, Wehrle R. Antagonism of serotonergic 5-HT2A/2C receptors: Mutual improvement of sleep, cognition, and mood? Eur J Neurosci. 2009;29:1795-1809.

36. Millan MJ, Gobert A, Lejeune F, et al. The novel melatonin agonist agomelatine is an antagonist at 5-hydoxytryptamine-2C receptors, blockade of which enhances the activity of frontocortical dopaminergic and adrenergic pathways. J Pharmacol Exp Ther. 2003;306:954-964.

37. Egerton A, Ahmad R, Hirani E, Grasby PM. Modulation of striatal dopamine release by 5-HT2A and 5-HT2C receptor antagonists: [11C]raclopride PET studies in the rat. Psychopharmacology. 2008; 200:487-496.

38. Shelton RC, Papakostas GI. Augmentation of antidepressants with atypical antipsychotics for treatment-resistant major depressive disorder. Acta Psychiatr Scand. 2008;117:253-259.

39. Quera-Salva MA, Vanier B, Laredo J, et al. Major depressive disorder, sleep EEG and agomelatine: An open-label study. Int $J$ Neuropsychopharmacol. 2007;10:691-696.

40. Kupfer DJ. Depression and associated sleep disturbances: Patient benefits with agomelatine. Eur Neuropsychopharmacol. 2006; 16(Suppl 5):S639-S643.

41. Leproult R, Van Onderbergen A, L'Hermite-Baleriaux M, Van Cauter E, Copinschi G. Phase-shifts of 24-h rhythms of hormonal release and body termperature following early evening administration of the melatonin agonist agomelatine in healthy older men. Clin Endocrinol. 2005;63:298-304

42. Descamps A, Rousset C, Millan M, Spedding M, Delagrange P, Cespuglio R. Influence of the novel antidepressant and melatonin agonist/serotonin $2 \mathrm{C}$ receptor antagonist, agomelatine, on the rat sleepwake cycle architecture. Psychopharmaology. 2009;2005:93-106.

43. Racagni G, Riva MA, Popoli M. The interaction between the internal clock and antidepressant efficacy. Int Clin Psychopharmacol. 2007; 22(Suppl 2):S9-S14

44. Duman RS, Monteggia LM. A neurotrophic model for stress-related mood disorders. Biol Psychiatry. 2006;59:1116-1127.

45. Sapolsky RM. Glucocorticoids and hippocampal atrophy in neuropsychiatric disorders. Arch Gen Psychiatry. 2000;57:925-935.

46. Wenk GL, Parsons CG, Danysz W. Potential role of N-methyl-Daspartate receptors as executors of neurodegeneration resulting from diverse insults: Focus on memantine. Behav Pharmacol. 2006;17: 411-424.

47. Heine VM, Maslam S, Zareno J, Joels M, Lucassen PJ. Suppressed proliferation and apoptotic changes in the rat dentate gyrus after acute and chronic stress are reversible. Eur J Neuroscience. 2004;19:131-144.

48. Einat H, Manji HK. Cellular plasticity cascades: Genes-to-behavior pathways in animal models of bipolar disorder. Biol Psychiatry. 2006; 59:1160-1171.

49. Banasr M, Soumier A, Hery M, Mocaer E, Daszuta A. Agomelatine, a new antidepressant, induces regional changes in hippocampal neurogenesis. Biol Psychiatry. 2007;59:1087-1096.

50. Gressens P, Schwendimann L, Husson I, et al. Agomelatine, a melatonin receptor agonist with 5-HT2C receptor antagonist properties, protects the developing murine white matter against excitotoxicity. Eur J Pharmacol. 2008;588:58-63. 
51. Barbiero VS, Zappettini S, Mocaer E, et al. The novel antidepressant agomelatine reduces release of glutamate and related presynaptic mechanisms in rat hippocampus. Eur Neuropyschopharmacol. 2007; 17(Suppl 4):S365.

52. Calabrese F, Pescara F, Racagni G, Mocaer E, Molteni R, Riva MA. Modulation of Arc and BDNF following acute and chronic administration of agomelatine in rats. Eur Neuropyschopharmacol. 2007; 17(Suppl 4):S333-S334.

53. Soumier A, Banasr M, Lortet S, et al. Mechanisms contributing to the phase-dependent regulation of neurogenesis by the novel antidepressant, agomelatine, in the adult rat hippocampus. Neuropsychopharmacology. DOI: $10.1038 / \mathrm{npp} .2009 .72$.

54. Conboy L, Tanrikut C, Zoladz PR, et al. The antidepressant agomelatine blocks the adverse effects of stress on memory and enables spatial learning to rapidly increase neural cell adhesion molecule (NCAM) expression in the hippocampus of rats. Int J Neuropsychopharmacol. 2009;12:329-341.

55. Paizanis E, Lelievre V, Saurini F, et al. Effects of agomelatine and fluoxetine on hippocampal cell survival, glucocorticoid receptors, and BDNF expression in a model of depression. Eur Neuropyschopharmacol. 2008;18(Suppl 4):S316.

56. Zupancic M, Guilleminault C. Agomelatine: A preliminary review of a new antidepressant. CNS Drugs. 2006;20:981-992.

57. Dolder CR, Nelson M, Snider M. Agomelatine treatment of major depressive disorder. Ann Pharmacother. 2008;42:1822-1831.

58. Loo H, Hale A, D'Haenen H. Determination of the dose of agomelatine, a melatoninergic agonist and selective 5-HT2C antagonist, in the treatment of major depressive disorder: A placebo-controlled dose range study. Int Clin Psychopharmacol. 2002;17:239-247.

59. Kennedy SH, Emsley R. Placebo-controlled trial of agomelatine in the treatment of major depressive disorder. Eur Neuropsychopharmacol. 2006;16(2):93-100.

60. Olie JP, Kasper S. Efficacy of agomelatine, a MT1/MT2 receptor agonist with 5-HT2C antagonistic properties, in major depressive disorder. Int J Neuropsychopharmacol. 2007;10:661-673.

61. Goodwin GM, Emsley R, Rembry S, Rouillon F; for the Agomelatine Study Group. Agomelatine prevents relapse in patients with major depressive disorder without evidence of a discontinuation syndrome: A 24-week randomized double-blind placebo-controlled trial. J Clin Psychiatry. In Press.

62. Kennedy SH, Rizvi S, Fulton K, Rasmussen J. A double-blind comparison of sexual functioning, antidepressant efficacy, and tolerability between agomelatine and venlafaxine XR. J Clin Psychopharmacol. 2008;28:329-333.

63. Montejo AL, Prieto N, Terleira A, et al. Better sexual acceptability of agomelatine ( $25 \mathrm{and} 50 \mathrm{mg}$ ) compared with paroxetine $(20 \mathrm{mg}$ ) in healthy male volunteers: An 8-week placebo-controlled study using the PRSEXDQ-SALSEX scale. J Psychopharmacol OnlineFirst. DOI:10.1177/0269881108096507 November 21, 2008.

64. Lemoine P, Guilleminault C, Alvarez E. Improvement in subjective sleep in major depressive disorder with a novel antidepressant agomelatine: Randomized double-blind comparison with venlafaxine. J Clin Psychiatry. 2007;68:1723-1732.

65. Wulff K, Bayle F, Hajak G, et al. Exploratory study of actigraphic and psychometric measures in depressed patients treated with agomelatine or sertraline (abstract). Eur Neuropsychopharmacol. 2007; 17(Suppl 4):S326.

Neuropsychiatric Disease and Treatment

\section{Publish your work in this journal}

Neuropsychiatric Disease and Treatment is an international, peerreviewed journal of clinical therapeutics and pharmacology focusing on concise rapid reporting of clinical or pre-clinical studies on a range of neuropsychiatric and neurological disorders. This journal is indexed on PubMed Central, the 'PsycINFO' database and CAS, and is the official
66. Kasper S, Laigle L, Bayle F. Superior antidepressant efficacy of agomelatine versus sertraline: A randomised double-blind study (abstract). Eur Neuropsychopharmacol. 2008;18(Suppl 4):S336-S337.

67. Montgomery SA, Kennedy SH, Burrows GD, Lejoyeux M, Hindmarch I. Absence of discontinuation symptoms with agomelatine and occurrence of discontinuation symptoms with paroxetine: A randomized, double-blind, placebo-controlled discontinuation study. Int Clin Psychopharmacol. 2004;19(5):271-280.

68. Stein DJ, Ahokas AA, de Bodinat C. Efficacy of agomelatine in generalized anxiety disorder: A randomized double-blind placebo-controlled study. J Clin Psychopharmacol. 2008;28:561-566.

69. Fabiano A, de Leersnyder H. Agomelatine efficacy on major sleep disturbances in Smith-Magenis syndrome: An exploratory open study in children (abstract). Eur Neuropsychopharmacol. 2007;17(Suppl 4):S567.

70. Wisniewski SR, Rush AJ, Nierenberg AA, et al. Can phase III trial results of antidepressant medications be generalized to clinical practice? A STAR*D report. Am J Psychiatry. 2009;166:599-607.

71. Howland RH, Thase ME. Mood disorders: Therapeutic armamentarium. In: D'haenen H, den Boer JA, Willner P, Eds. Biological Psychiatry. London, England: John Wiley \& Sons, Ltd; 2002:861-875.

72. Howland RH. Medication adherence. Psychiatr Ann. 2008;38:323-326.

73. Howland RH. Limitations of evidence in the practice of evidence-based medicine. Psychiatr Ann. 2008;38:334-336.

74. Wisniewski SR, Fava M, Trivedi MH, et al. Acceptability of second-step treatments to depressed outpatients: A STAR*D report. Am J Psychiatry. 2007; 164:753-760.

75. Loo H, Dalery J, Macher JP. Pilot study comparing in blind the therapeutic effect of two doses of agomelatine, melatoninergic agonist and selective $5 \mathrm{HT} 2 \mathrm{C}$ receptors antagonist, in the treatment of major depressive disorders [article in French]. Encephale. 2002;28:356-362.

76. Howland RH. Evaluating the safety of medications during pregnancy and lactation. J Psychosoc Nurs Ment Health Serv. 2009;47:19-22.

77. Howland RH. Prescribing psychotropic medications during pregnancy and lactation: Principles and guidelines. J Psychosoc Nurs Ment Health Serv. 2009;47:19-23.

78. Trivedi MH, Rush AJ, Gaynes BN, et al. Maximizing the adequacy of medication treatment in controlled trials and clinical practice: STAR*D measurement-based care. Neuropsychopharmacology. 2007;32:2479-2489.

79. Howland RH. An overview of seasonal affective disorder and its treatment. The Physician and Sportsmedicine. In press.

80. Pandi-Perumal SR, Moscovitch A, Srinivasan V, Spence DW, Cardinali DP, Brown GM. Bidirectional communication between sleep and circadian rhythms and its implications for depression: Lessons from agomelatine. Prog Neurobiol. 2009;88:264-271.

81. Calabrese JR, Guelfi JD, Perdrizet-Chevallier C. Agomelatine adjunctive therapy for acute bipolar depression: Preliminary open data. Bipolar Disord. 2007;9:628-635.

82. Pjrek E, Winkler D, Konstantinidis A, Willeit M, Praschak-Rieder N, Kasper S. Agomelatine in the treatment of seasonal affective disorder. Psychopharmacology. 2007;190:575-579.

83. Howland RH. Current research in treatment-resistant depression: When to augment and when to switch. MedscapeCME Psychiatry and Mental Health. Published Online July 23, 2009, URL: http://cme.medscape. com/viewarticle/705917.

84. Howland RH. Understanding the clinical profile of a drug on the basis of its pharmacology: Mirtazapine as an example. J Psychosoc Nurs Ment Health Serv. 2008;46:19-23.

journal of The International Neuropsychiatric Association (INA). The manuscript management system is completely online and includes a very quick and fair peer-review system, which is all easy to use. Visit http://www.dovepress.com/testimonials.php to read real quotes from published authors. 